\title{
DIFERENCIAS EN EL FUNCIONAMIENTO NEUROCOGNITIVO E IMPULSIVIDAD RASGO SEGÚN EL PATRÓN DE CONSUMO DE ALCOHOL EN JÓVENES DE 18 A 30 AÑOS
}

\section{DIFFERENCES IN NEUROCOGNITIVE FUNCTIONING AND IMPULSIVITY ACCORDING TO PATTERN OF ALCOHOL CONSUMPTION IN 18- TO 30- YEARS-OLD YOUTH}

Ignacio Acuña, Yanina Michelini, Juan Ignacio Guzmán y Juan Carlos Godoy

CIPSI Grupo Vinculado CIECS-UNC-CONICE, Argentina

\section{Abstract}

Introduction. Adolescence is a period of life characterized by impulsive behavior and risky decision making (DM) which is associated with onset and progression in alcohol consumption. Aim. Assess the effect of the pattern of alcohol consumption (PAC) over DM executive function (EF) and impulsivity in an 18- to 30-year-old college sample. Methods. A prospective ex-post facto design was used to assess PAC effect over DM and impulsivity. IGT, GDT, and CBT were used to assess DM, WCST to assess executive function and UPPS-P sub-scales to assess impulsivity. Results. Participants classified as Binge and Heavy drinkers had the worst performance on IGT and CBT and were higher in all dimensions of UPPS-P, except to Positive Urgency. Additionally, they have less correct answers on WCST. Conclusion. This data allows to conclude severe PAC has effects on neurocognitive performance in young.

Keywords: decision making, impulse behavior, young, alcohol drinking

\section{Resumen}

Introducción. La adolescencia es un período caracterizado por las conductas impulsivas y la toma de decisiones (TD) arriesgadas lo cual se asocia al inicio y la progresión del consumo de alcohol y de otras drogas. Objetivo: evaluar el efecto del patrón de consumo de alcohol (PCA) sobre la TD, la función ejecutiva (FE) y la impulsividad rasgo en una muestra de estudiantes universitarios de 18 a 30 años. Metodología. Se usó un diseño ex post facto prospectivo para evaluar el efecto del consumo de alcohol en la TD y en la impulsividad rasgo. Para evaluar la TD se utilizó la IGT, la GDT y la CBT y, para evaluar FE, la WCST. Para medir la impulsividad rasgo se consideraron los puntajes en las sub-escalas de la Escala UPPS-P. Resultados. Los participantes clasificados como PCA intensivo y PCA pesado tuvieron peor rendimiento en las tareas IGT y CBT y, además, puntuaron más alto en todas las dimensiones de impulsividad medidas con la UPPS-P, excepto en Urgencia Positiva. Adicionalmente, tuvieron menos respuestas correctas en la WCST. Conclusión. Los PCA más severos se asocian con un rendimiento neurocognitivo más pobre en jóvenes argentinos de 18 a 30 años de edad.

Palabras clave: toma de decisiones, comportamiento impulsivo, jóvenes, consumo de bebidas alcohólicas. 
La comprensión de las características del desarrollo de adolescentes y jóvenes ha mejorado significativamente gracias a los aportes de la Psicología Cognitiva y de las Neurociencias. En efecto, desde la caracterización de la adolescencia como un periodo de "tormenta y tensión" que presentara Stanley Hall (1904), pasando por la proposición de una "adultez emergente" (Arnett, 2000), se han ido desarrollando miradas más complejas sobre los cambios que experimentan adolescentes y jóvenes. En ese sentido, por ejemplo, cabe mencionar los diferentes modelos de organización del cerebro adolescente y joven que se agrupan bajo la denominación de Modelos de Sistemas Duales (ver, por ejemplo: Casey, Getz, \& Galván, 2008; Shulman et al., 2016). Estos modelos consideran que hay un desarrollo asimétrico entre las estructuras encargadas del procesamiento y del control cognitivo, y aquellas encargadas del procesamiento de información socioemocional (Steinberg, 2008).

Esta disparidad en el tiempo de maduración de ambos conjuntos de estructuras cerebrales explicaría por qué los adolescentes son capaces de razonar y considerar lógicamente las consecuencias de sus acciones, pero llegado el momento de inhibir la búsqueda de reforzamiento inmediato (emocionalmente hablando) no son capaces de lograrlo y es recién en los primeros años de la juventud (20/25 años) cuando el sistema de control socioemocional y control cognitivo empiezan a funcionar de manera integrada (Shulman et al., 2016). Como correlato neurobiológico de la particular interacción entre el sistema de control cognitivo y el sistema de control socioemocional, varias áreas cerebrales continúan desarrollándose durante la adolescencia y la juventud, especialmente la corteza prefrontal, que está íntimamente asociada a las Funciones Ejecutivas (FE), la Toma de Decisiones (TD) y el control inhibitorio de las respuestas impulsivas (Albert \& Steinberg, 2011; Blakemore \& Robbins, 2012).

Aun así, durante las fases finales de la adolescencia (a partir de los 18 años) los adolescentes tienen menor predisposición biológica para tomar riesgos, pero también tienen mayor probabilidad de involucrarse en este tipo de conductas. Esto, en parte, debido al aumento de estímulos potencialmente riesgosos provenientes del mundo real como, por ejemplo, la disponibilidad financiera, la residencia fuera del núcleo familiar, la edad legal para beber y conducir, entre otros (Shulman et al.,
2016). En este sentido, el uso exploratorio de alcohol y otras sustancias, la toma de decisiones riesgosas y la búsqueda de sensaciones serían comportamientos esperables aún en los primeros años de la segunda década de vida (Spear, 2013).

Ciertamente, las conductas de consumo de alcohol de los adolescentes y jóvenes se encuentran frecuentemente asociadas al comportamiento impulsivo y a la toma de decisiones desadaptativas (Acuña, Castillo, Bechara, \& Godoy, 2013; Parada et al., 2012). Al respecto, cabe destacar que en Argentina la edad de inicio de consumo se ubica entre los 12 y 13 años (Pilatti, Brussino, \& Godoy, 2013; Pilatti, Godoy, Brussino, \& Pautassi, 2013). Mientras que, por otro lado, la Organización Mundial de la Salud (2014) reporta que del total de adolescentes que consumen alcohol, el 16\% tiene Consumo Episódico Elevado (CEE), lo que equivale a 60 gramos puros de alcohol por ocasión de bebida con una frecuencia, al menos, mensual. Se ha sugerido que el pico de consumo de alcohol que se da hacia el final del periodo adolescente podría deberse a la impulsividad que presentan los adolescentes en su etapa media. Ello mantendría la escalada en el consumo de alcohol y afectaría la maduración de los controles cognitivos de la conducta (Albert \& Steinberg, 2011; Quinn \& Harden, 2013).

En este contexto, el Consumo Intensivo de Alcohol ( $\mathrm{ClA}$; binge drinking en inglés) es un patrón de consumo muy frecuente entre adolescentes y jóvenes que se caracteriza por ingestas intermitentes en forma de atracones, concentradas en sesiones de pocas horas, principalmente durante las noches de fin de semana y en grupos de pares (National Institute on Alcohol Abuse and Alcoholism, 2013; Parada et al., 2011). Un estudio de revisión reciente (Spear, 2015) muestra que los mayores índices de CIA se dan en la adolescencia tardía (18 a 25 años). Además, un CIA durante la adolescencia y la juventud podría predecir una mayor vulnerabilidad a los efectos del alcohol en la adultez pudiendo generarse una dependencia al alcohol (Pilatti, Caneto, Garimaldi, Vera, \& Pautassi, 2014).

En relación a la toma de decisiones se la entiende como la capacidad para seleccionar un curso de acción entre un conjunto de posibles alternativas conductuales (Acuña et al., 2013; Martínez-Selva, Sánchez-Navarro, Bechara, 2006). En ese sentido, se ha observado que adolescentes y jóvenes que presentan un consumo 
elevado de alcohol pueden experimentar, como consecuencia, fallas en la TD (Goudriaan, Grekin, \& Sher, 2007). Al respecto, estudios experimentales con adolescentes y jóvenes universitarios realizados en el ámbito local, mostraron que los jóvenes intoxicados de forma aguda con alcohol tuvieron un patrón de toma de decisiones significativamente más desventajoso que los no intoxicados (Acuña et al., 2013). En otro estudio, los estudiantes identificados como consumidores intensivos de alcohol presentaron un estilo de toma de decisiones independiente del contexto, lo cual se asocia con decisiones que no se basan en las contingencias de recompensa y castigo sino que estarían en relación a la mera satisfacción inmediata (Michelini, Acuña, \& Godoy, 2016).

Por otro lado, también es posible considerar que los déficits en la TD sean una de las causas del consumo abusivo de sustancias. Al respecto, algunos estudios señalan que individuos con problemas en su capacidad de TD, la mayoría de las veces, no pueden resistirse al acceso a las sustancias de abuso disponibles en su entorno (Bechara, 2005; Goldstein \& Volkow, 2002). De esta manera, es posible que los adolescentes y jóvenes con un consumo de alcohol que en principio es casual y no problemático avancen hacia un consumo compulsivo y descontrolado (Xiao et al., 2013). Sin embargo, más allá de esta observación, en este estudio se consideró el patrón de consumo de alcohol como la variable independiente $y$, por lo tanto, el desempeño en pruebas de TD como la variable dependiente de interés. Esto va en la línea de otros estudios que han señalado que el consumo de alcohol y de otras sustancias impacta sobre diversos dominios neurocognitivos en adolescentes y jóvenes. Por ejemplo, un estudio en el cual se vigiló por 10 años el consumo de alcohol y otras sustancias además del desempeño en tareas cognitivas de un grupo adolescentes y jóvenes, reveló que el consumo pesado de alcohol durante el periodo analizado afectó el rendimiento cognitivo, sobre todo las habilidades visuoespaciales, de aprendizaje verbal y de memoria (Hanson, Medina, Padula, Tapert, \& Brown, 2011). A su vez, en otro estudio experimental, estudiantes universitarios con un patrón de consumo intensivo de alcohol tuvieron un peor rendimiento en la prueba de memoria de trabajo Span de dígitos reversa y en la prueba de Función Ejecutiva (FE) Wisconsin Card Sorting Test, en comparación con los estudiantes que no consumían alcohol. Además, los varones con CIA tuvieron un peor rendimiento en memoria de trabajo respecto de las mujeres CIA y las mujeres no CIA (Parada et al., 2012).

En este sentido, también se ha reportado que la FE se ve afectada por el consumo de alcohol de los jóvenes. Los estudiantes universitarios con CIA mostraron un rendimiento más pobre en diversos índices de $\mathrm{FE}$ comparados con los que no tenían CIA o eran abstemios. Estos reportes coinciden en que el efecto del consumo abusivo de alcohol en el rendimiento en medidas dependientes del funcionamiento de las distintas áreas de la corteza prefrontal está relacionado con que estas áreas siguen desarrollándose hasta ya iniciada la tercera década de vida y que el consumo de alcohol podría estar afectando el desarrollo normal de estas estructuras (Crews, He, \& Hodge, 2007; Mota et al., 2013; Parada et al., 2012).

Otra variable de interés para este trabajo es la impulsividad, que puede ser considerada desde un punto de vista conductual (Rogers, Moeller, Swann, \& Clark, 2010) o, como en este caso, como un rasgo de personalidad. En este último sentido es entendida como un constructo multidimensional que integra varios rasgos de personalidad como la falta de premeditación, la falta de perseverancia, la búsqueda de sensaciones, y la urgencia (Cyders, 2013). Desde un abordaje neurocognitivo, la impulsividad puede ser equiparada con el término desinhibición, en referencia a la idea de un mecanismo de control de "arriba hacia abajo" que normalmente suprime las respuestas automáticas o de búsqueda de recompensa que no son apropiadas a las demandas actuales (Aron, 2007). Este mecanismo de control puede ser interrumpido tras una lesión en el cerebro o por alguna forma de trastorno funcional resultando en una predisposición hacia actos impulsivos. Definida de esta manera, la impulsividad tiene una clara importancia para los trastornos por consumo de sustancias. En efecto, en numerosos estudios la impulsividad aparece como factor predictor y también como efecto del CIA en adolescentes y jóvenes (Coskunpinar, Dir, \& Cyders, 2013; Stautz \& Cooper, 2013). Al respecto, estudios del ámbito local han revelado que la impulsividad y el consumo concomitante de alcohol afectan el rendimiento en tareas de toma de decisiones, lo que podría incidir a su vez sobre la progresión en el consumo de la sustancia (Caña, Michelini, Acuña, \& Godoy, 2015). Por otra parte, también la impulsividad puede resultar afectada por el uso agudo 
y/o crónico de alcohol o de otras sustancias (de Wit, 2009). Por ejemplo, algunos estudios de trayectorias indican que los cambios, tanto sean de incremento como de disminución del consumo de alcohol durante la adolescencia y la juventud, se acompañan de modificaciones de la conducta impulsiva, en el mismo sentido (Littlefield, Sher, \& Steinley, 2010; White et al., 2011).

Así, este trabajo tuvo como objetivo evaluar si los adolescentes y jóvenes de 18 a 30 años de edad con distinto Patrón de Consumo de Alcohol (PCA) difieren en su desempeño en pruebas de TD, en la función ejecutiva (FE) y en la impulsividad como rasgo de personalidad. La hipótesis de trabajo fue que aquellos participantes que presentaran un PCA más severo, mostrarían un peor rendimiento en las tareas de TD, de FE y tendrían puntuaciones más altas en escalas de impulsividad rasgo.

\section{MÉTODO}

\section{Diseño}

Se utilizó un diseño ex post facto prospectivo 3 (PCA Liviano, Intensivo y Pesado) x 2 (Sexo Masculino y Femenino) (Montero \& León, 2007) para evaluar el efecto del patrón de consumo de alcohol sobre el desempeño en las pruebas de TD y sobre las respuestas en las subescalas de Impulsividad rasgo.

\section{Participantes}

La muestra se compuso de 217 estudiantes de la Universidad Nacional de Córdoba (64,5\% mujeres) entre 18 y 30 años de edad $(M=23,22$, DS $\pm 2,44)$, voluntarios, con 12 a 18 años de educación formal $(M=14,60$, DS= , \pm 110 ) que dieron su consentimiento por escrito. Los participantes no debían tener ni haber tenido alguna alteración neurológica o psiquiátrica ni estar bajo tratamiento por dichas afecciones, no poseer alguna adicción o estar bajo tratamiento por adicción. Estos datos fueron relevados mediante auto-reporte (Wood, Sher, \& Rutledge, 2007). Se les informó que podían abandonar el estudio en cualquier momento, y se les aseguró que los datos solo se utilizarían con fines estadísticos y permanecerían en anonimato.

\section{Instrumentos}

Cuestionario sociodemográfico: se incluyeron preguntas sobre edad, sexo, nivel educativo, estado civil, historia familiar de consumo, padecimiento de enfermedades y/o tratamientos.

Cuestionario de patrón de consumo de alcohol y otras sustancias: con los siguientes indicadores de consumo: frecuencia, cantidad, frecuencia de consumo hasta la ebriedad e intención de consumo en el próximo fin de semana, semana y mes. Además, indaga sobre el uso de tabaco y otras sustancias (Caña et al., 2015).

Wisconsin Card Sorting Test (WCST; Grant \& Berg, 1948): se utilizó la versión electrónica de 128 cartas de la prueba. En esta prueba se le pide al participante que empareje las cartas que aparecen en el mazo de abajo, con uno de los cuatro mazos que aparecen arriba, haciendo clic debajo de la carta con la que cree que coincide. Cuando el participante realiza diez clasificaciones correctas consecutivas, consigue una categoría, y se cambia el criterio de clasificación sin previa advertencia. Además, se le menciona que se le señalará si lo hizo de manera correcta o incorrecta y que, de acuerdo con esa información, debe tratar de obtener tantas cartas correctas como sea posible. El participante debe clasificar primero por color, luego por forma y luego por número. El procedimiento continúa hasta que se han completado 6 categorías correctas o hasta que se han colocado las 128 cartas (Heaton, Chelune, Talley, Kay, \& Curtis, 1993).

Game of Dice Task (GDT; Brand et al., 2005): la tarea fue diseñada para medir la influencia de la FE en la TD en situaciones de apuestas. A los participantes se les solicita que incrementen su capital ficticio inicial (\$1.000) dentro de los 18 tiros del dado. Las reglas y monto de ganancias y pérdidas son explícitamente visualizadas. Además, el número de tiros remanentes es también mostrado en la pantalla. La puntuación se obtiene clasificando las elecciones de uno y dos números como arriesgada o desventajosa, respectivamente, mientras que las opciones de tres y cuatro números como no arriesgada y ventajosa respectivamente. Al total de elecciones de tres y cuatro números se resta el total de elecciones de uno y dos números.

lowa Gambling Task (IGT; Bechara, Damasio, Damasio, \& Anderson, 1994): es una prueba que evalúa la capacidad para tomar decisiones ventajosas o desventajosas. 
Consta de 4 mazos de cartas. En los desventajosos (A y B), las elecciones conllevan una alta ganancia y una alta penalización. En los mazos ventajosos (C y D), la ganancia inmediata y las pérdidas futuras son menores. Para puntuar la tarea, se suma el total de cartas elegidas de los mazos A y B y se resta este resultado al total de cartas elegidas de los mazos C y D; el resultado da un puntaje neto para el total de la prueba.

Cognitive Bias Task (CBT; Goldberg, Harner, Lovell, Podell, \& Riggio, 1994): es una prueba de respuesta múltiple en la que se evalúa el estilo en la TD. Cada ensayo consiste en la presentación de un estímulo clave y luego 2 elecciones posibles. Se mide el índice de similitud de acuerdo con el tipo de elecciones que hace el participante con relación al estímulo clave. Los índices de similitud son sumados a lo largo de los ensayos para generar una puntuación que va de 80 a 220. Una puntuación baja indica un patrón de elección diferente del estímulo clave. Una puntuación alta indica elecciones similares al estímulo clave y una puntuación media indica elecciones alternantes sin relación con el estímulo clave o un número equivalente de elecciones diferentes o similares.

Cuestionario de Impulsividad UPPS-P (Whiteside $y$ Lynam, 2001, en Verdejo-García, Lozano, Moya, Alcázar, \& Pérez-García, 2010): Se utilizó la adaptación española. Mide cinco aspectos de la personalidad que llevan a la conducta impulsiva: la urgencia negativa, la (falta de) perseverancia, la (falta de) premeditación, la búsqueda de sensaciones, y la urgencia positiva. Presenta valores adecuados de confiabilidad para toda la escala ( $\alpha=0.94)$.

Escala L.: es una de las cuatro escalas que componen el Cuestionario de Personalidad de Eysenck (EPQ) y mide la tendencia al falseamiento positivo, es decir, al disimulo (Eysenck \& Eysenck, 1997). Consta de diez preguntas dicotómicas que se puntúan con 0 o 1 de acuerdo a una grilla preestablecida. Aquellos participantes que presenten \pm 2 desviaciones estándar de su grupo deben ser considerados como participantes con alta tendencia al falseamiento positivo. En esos casos, la decisión es la de no incluir su información en el estudio.

\section{Procedimiento}

La investigación se llevó a cabo completamente en las instalaciones de la Facultad de Psicología de la UNC en una sala que se mantuvo constante a lo largo de todo el estudio. La evaluación de cada participante tuvo una duración de 120 minutos y fue realizada por los responsables de este estudio y colaboradores entrenados. Primero, se informó a cada uno de los participantes acerca de las condiciones en que se desarrollaba la investigación, las mismas fueron presentadas por escrito junto con la declaración de consentimiento informado. Este documento debió ser firmado por los participantes. Luego, los participantes recibieron un cuestionario con la finalidad de recabar datos sociodemográficos. A continuación, se procedió a leer y mostrar a través de la pantalla de la computadora las instrucciones de la IGT. Luego se administró la CBT y finalmente, la WCST. Una vez finalizadas estas pruebas, se le indicó al participante que contaba con 10 minutos de descanso, que no podía fumar y que tenía agua disponible si lo deseaba. Transcurrido este período de descanso, se aplicó la GDT. Luego se administró el cuestionario UPPS-P y finalmente se aplicó la Escala L. Esta última fue incluida a los fines de controlar el efecto de la deseabilidad social en las respuestas relacionadas al consumo de alcohol y sustancias. Al respecto, cabe señalar que ninguno de los participantes obtuvo puntuaciones mayores a \pm 2 desviaciones estándar. El orden de las pruebas fue el mismo para todos los participantes, el cual se estableció según el tiempo de ejecución de cada prueba y cuidando que la realización de una prueba no afectara el desempeño en la siguiente.

\section{Análisis de datos}

Para realizar el Análisis de Clases Latentes, necesario para la conformación de los grupos en función del consumo de alcohol, se usó LatentGold 4.5. Para la comparación del desempeño en las pruebas de FE y TD, así como en las puntuaciones en Impulsividad rasgo entre los distintos grupos de bebedores de alcohol se corrieron Análisis de Varianza (ANOVA) Factorial y de Medidas Repetidas (cuando fue posible), y en caso de ser necesario se utilizó la prueba post hoc de Diferencias Estadísticas Mínimas (LSD por sus siglas en inglés). Estos análisis fueron realizados con SPSS Statistics 17.0 y STATISTICA 8. En todos los casos el nivel de significación estadística se fijó en $p<.05$.

\section{Análisis de Clases Latentes}

El Patrón de Consumo de Alcohol (PCA) de cada participante se determinó con un Análisis de Clases Latentes (LCA, por sus siglas en inglés, Latent Class 
Analysis). Para ello se utilizaron los siguientes indicadores de consumo: frecuencia, cantidad, frecuencia de consumo hasta la ebriedad e intención de consumo en la próxima semana. Así, se probaron modelos de 1 a 5 clases latentes con el fin de lograr el modelo más parsimonioso que ofreciera un buen ajuste a los datos y cumpliera con el supuesto de independencia local. Los modelos fueron comparados en función de tres medidas que consideran la bondad de ajuste y la parsimonia: el Criterio de Información Bayesiana (BIC), el Criterio de Información de Akaike (AIC) y el Criterio de Información de Akaike 3 (AIC3). En estas medidas, valores más bajos indican un mejor ajuste del modelo. Dos de los tres indicadores utilizados (BIC y AIC3) dieron cuenta que el modelo de tres clases latentes era el que presentaba mejor ajuste. El error de clasificación en este modelo fue del 7\%. En el modelo elegido no se observó dependencia local. La Tabla 1 refleja los indicadores de bondad de ajuste de los modelos evaluados. Y se verificó el aporte de cada indicador a la discriminación entre clases mediante el estadístico de Wald, todos los indicadores resultaron ser significativos para diferenciar las clases (Tabla 2).

Tabla 1. Indicadores de bondad de ajuste de los cinco modelos analizados

\begin{tabular}{lcccc}
\hline & BIC(LL) & AIC(LL) & AIC3(LL) & $\begin{array}{c}\text { Error de } \\
\text { Clasificación }\end{array}$ \\
\hline 1Clase & 1747,568 & 1713,632 & 1723,632 & 0 \\
2Clases & $\mathbf{1 6 5 6 , 2 9 3}$ & 1605,389 & 1620,389 & 0,069 \\
3Clases & 1657,866 & $\mathbf{1 5 8 8 , 9 6 9}$ & $\mathbf{1 6 0 9 , 9 9 4}$ & $\mathbf{0 , 0 7 8}$ \\
4Clases & 1673,810 & 1589,994 & 1613,969 & 0,161 \\
5Clases & 1686,844 & 1585,035 & 1615,035 & 0,129
\end{tabular}

Nota: $\mathrm{BIC}=$ Criterio de Información Bayesiana; $\mathrm{AIC}=$ Criterio de Información de Akaike; $\mathrm{AIC}=$ = Criterio de Información de Akaike 3
Tabla 2. Análisis de la contribución de cada uno de los indicadores al modelo de clases latentes

\begin{tabular}{lccccc}
\hline & Clase1 & Clase2 & Clase3 & Wald & $p$ \\
\hline & & & & & \\
Tragos & 0,209 & $-1,113$ & 0,904 & 17,900 & 0,000 \\
Frecuencia & $-0,459$ & $-2,662$ & 3,121 & 30,218 & $2,70 \mathrm{E}-07$ \\
$\begin{array}{l}\text { Frecuencia } \\
\text { problema }\end{array}$ & $-1,450$ & $-2,801$ & 4,251 & 32,534 & $8,60 \mathrm{E}-08$ \\
$\begin{array}{l}\text { Intención } \\
\text { de consumo } \\
\text { semanal }\end{array}$ & 1,261 & $-1,669$ & 0,409 & 25,401 & $3,00 \mathrm{E}-06$ \\
& & & & & \\
\hline
\end{tabular}

A continuación, se estudió la probabilidad de respuesta en los distintos indicadores en cada una de las clases resultantes. La clase 1 se compuso por el 52\% de la muestra, caracterizándose por una alta probabilidad de consumir con una frecuencia semanal $(0,75)$, de beber una cantidad de alcohol aproximadamente de 1 a 5 tragos por ocasión de bebida $(0,57)$, y de tener un consumo problemático de más de 5 tragos mensualmente $(0,76)$, presentando una intención de consumo de alcohol afirmativa en la próxima semana muy elevada $(0,87)$. A esta clase se le designó como patrón de consumo "intensivo". La clase 2 comprendió el $31 \%$ de la muestra, presentó una alta probabilidad de consumir con frecuencia mensual $(0,61)$ y de beber entre 1 y 4,99 tragos $(0,85)$, manifestando no beber más que esa cantidad reportada por ocasión de bebida $(0,52)$, y sin intención de consumir la próxima semana $(0,66)$, por lo que se denominó esta clase como patrón de consumo "liviano". Por último, la clase 3 tuvo una prevalencia estimada del 15\%, presentó mayor probabilidad de beber con una frecuencia semanal $(0,93)$, una cantidad de 6 a $11,99(0,44)$, presentando un consumo problemático semanal $(0,92)$, y mayor probabilidad de intención de consumo en la categoría "sí, es muy posible" $(0,71)$. Esta clase de consumo se denominó "pesados". Los resultados se presentan en la Tabla 3. 
Tabla 3. Probabilidad de las clases y probabilidad de respuestas del modelo de 3 clases de consumo de alcohol

\begin{tabular}{|c|c|c|c|}
\hline & Intensivo & Liviano & Pesados \\
\hline Tamaño de la clase & 0,5286 & 0,3134 & 0,158 \\
\hline \multicolumn{4}{|l|}{ Tragos } \\
\hline 1 a 5,99 & 0,5783 & 0,8543 & 0,3696 \\
\hline 6 a 11,99 & 0,3511 & 0,1383 & 0,4495 \\
\hline Más de 12 & 0,0705 & 0,0074 & 0,1808 \\
\hline \multicolumn{4}{|l|}{ Frecuencia } \\
\hline Anual & 0,0068 & 0,1626 & 0 \\
\hline Mensual & 0,2339 & 0,6167 & 0,0081 \\
\hline Semanal & 0,7581 & 0,2207 & 0,9385 \\
\hline Diario & 0,0012 & 0 & 0,0534 \\
\hline \multicolumn{4}{|l|}{ Frecuencia Problema } \\
\hline No tomo & 0,0909 & 0,5284 & 0 \\
\hline Anual & 0,1136 & 0,1709 & 0 \\
\hline Mensual & 0,7642 & 0,2975 & 0,0755 \\
\hline Semanal & 0,0313 & 0,0032 & 0,9244 \\
\hline \multicolumn{4}{|l|}{ Intensión Semanal } \\
\hline No o es poco posible & 0,0161 & 0,6614 & 0,0727 \\
\hline Es Posible & 0,1076 & 0,236 & 0,2074 \\
\hline Si o es muy posible & 0,8763 & 0,1026 & 0,7199 \\
\hline
\end{tabular}

Sólo se retuvieron aquellos casos donde se obtuvo una probabilidad de clasificación mayor al 60\%. Así, se clasificaron a 218 casos, quedando sólo 1 caso sin clasificar.

\section{RESULTADOS}

En la WCST, se encontró un efecto principal del PCA de los participantes en el total de respuestas correctas, $F$ $(2,158)=4,593, \mathrm{p}<.05, \eta p 2=0,055$ (Figura 1). La prueba post hoc LSD, mostró que los participantes con un consumo liviano tuvieron una puntuación significativamente más baja que los bebedores intensivos $(p<.01)$. No se observaron diferencias estadísticamente significativas al comparar a los bebedores pesados con los bebedores intensivos, ni tampoco al compararlos con los bebedores livianos. Las otras medidas dependientes del WCST, no mostraron efectos significativos asociados al PCA y al sexo.

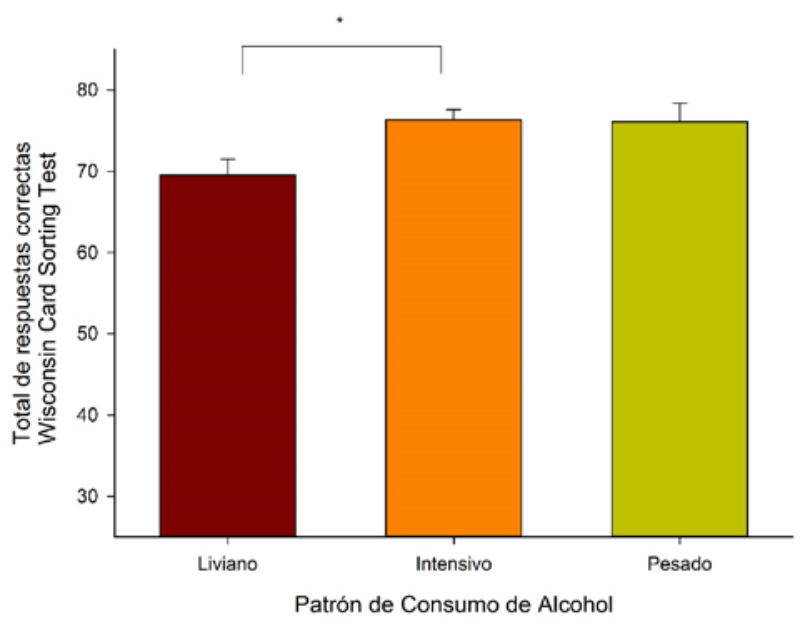

Figura 1. Total de respuestas correctas en WCST según PCA (error estándar de la media)

En relación a la GDT, se encontró un efecto significativo del PCA en las puntuaciones de los participantes $F(2,213)=4,307 p<.01, \eta p 2=0,039$ (Figura 2). La prueba post hoc LSD, mostró que los bebedores pesados tuvieron puntuaciones significativamente más bajas que los bebedores intensivos $(p<.01)$ y que los bebedores livianos $(p=<.01)$.

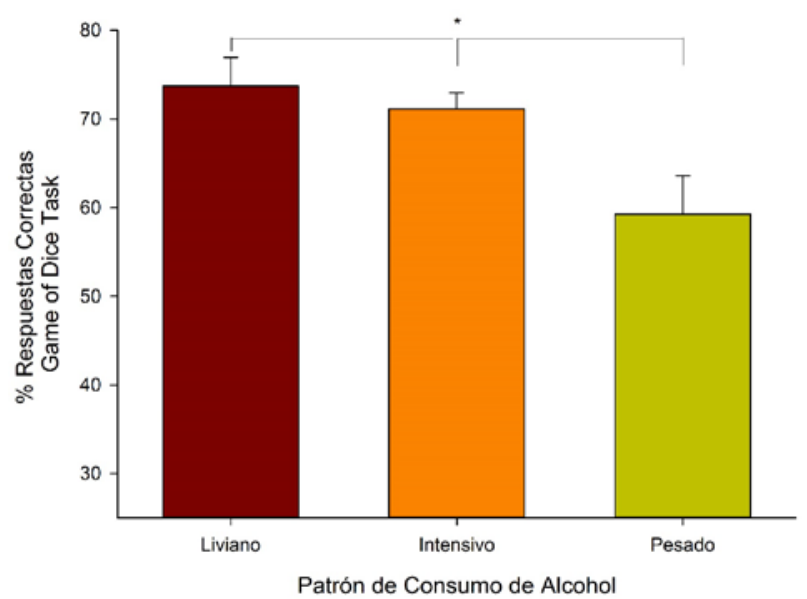

Figura 2. Porcentaje de respuestas correctas en GDT según PCA (error estándar de la media)

En la CBT, no se encontraron resultados significativos al analizar el puntaje global de la prueba. Un ANOVA de medidas repetidas mostró una interacción entre los bloques de la CBT y el PCA. Debido a que no fue posible asumir la esfericidad de los datos, se consideró la corrección de Greenhouse-Geisser (Tabachnick \& Fidell, 2007), que dio como resultado $F(2.751,585)=3,592$ $\mathrm{p}<.001, \eta p 2=0,033$ (Figura 3). La prueba LSD, en una comparación intra-grupo, mostró que los bebedores intensivos, en el bloque 1, hicieron elecciones más 
dependientes del contexto que en el bloque $2(p<.001)$, y que en el bloque $4(p<.05)$. El rendimiento de los consumidores livianos en el bloque 1 , no fue diferente del bloque 2, pero sí se diferenció significativamente con el bloque $3(p<.05)$, y con el bloque $4,(p<.05)$. Por su parte, los consumidores pesados, en el bloque 2 , realizaron elecciones más independientes del contexto que en el bloque $4(p<.001)$.

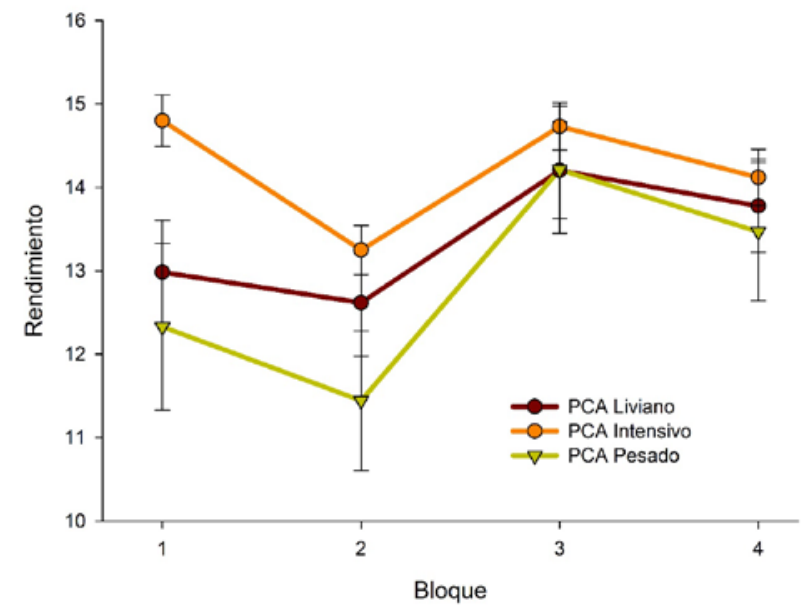

Figura 3. Rendimiento en CBT por bloques según PCA

También, se realizó una comparación inter-grupo (PCA intensivo vs. liviano vs. pesado), donde se encontraron diferencias significativas en tres de los cuatro bloques (Figura 4). Así, en el bloque 1 se observó que los bebedores intensivos hicieron elecciones más dependientes del contexto que los bebedores pesados $(p<.01)$. Entre bebedores livianos y pesados del mismo bloque no se encontraron diferencias significativas. En el bloque 2, se encontró una diferencia significativa entre los bebedores intensivos y los bebedores pesados, donde los intensivos hicieron elecciones más dependientes del contexto que los pesados $(p<.01)$. En el bloque 3 , se observó una diferencia significativa entre bebedores intensivos y pesados, donde los pesados realizaron elecciones más independientes del contexto que los intensivos $(p<.01)$. En el bloque 4 , no se encontraron efectos estadísticamente significativos. De la misma manera, se analizaron la puntuación total y por bloques de la IGT y no se encontraron diferencias significativas para ninguno de los factores individualmente ni en interacción.

Para la escala UPPS-P se realizó un ANOVA para cada dimensión. Así, para la Urgencia Negativa se encontró un efecto significativo del PCA, F $(2,213)=4,411$ $\mathrm{p}<.05, \eta p 2=0,040$ (Figura 4), la prueba LSD mostró que los bebedores pesados puntuaron más alto que los livianos $(p<.05)$, y a su vez, los bebedores livianos puntuaron más bajo que los intensivos $(p<01)$. No se encontraron diferencias significativas entre los consumidores pesados y los intensivos. Tampoco se encontraron diferencias significativas con respecto al sexo de los participantes ni para la interacción de sexo y PCA.

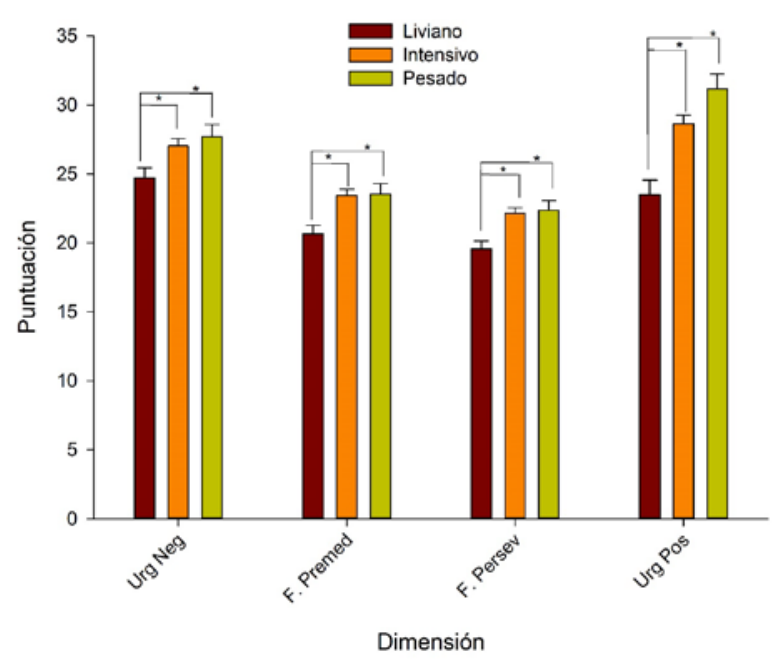

Figura 4. Puntuación en dimensiones de la UPPS-P según PCA

En la dimensión Falta de Premeditación se encontraron diferencias significativas para el PCA $F$ $(2,213)=7,227 \mathrm{p}<.001, n p 2=0,064$ (Figura 4), el análisis post hoc mostró que los bebedores pesados tuvieron puntuaciones más altas que los livianos $(p<.01)$, y estos últimos presentaron puntuaciones más bajas que los consumidores intensivos $(p<.001)$. No se encontraron diferencias significativas para el factor sexo, ni para la interacción de ambos factores.

Para la dimensión Falta de Perseverancia se encontró un efecto significativo del patrón de consumo $\mathrm{F}$ $(2,213)=7,657 \mathrm{p}<.001, n p 2=0,067$ (Figura 4). La prueba LSD muestra una diferencia entre los participantes con consumo pesado respecto de los bebedores livianos, puntuando los primeros más alto que los últimos, $(p<.01)$, mientras que los bebedores livianos tuvieron menores puntuaciones que los intensivos $(p<.01)$. No se encontraron diferencias significativas con respecto al sexo de los participantes ni para la interacción de sexo y PCA.

En el caso de la dimensión Búsqueda de Sensaciones se encontró un efecto significativo en 
función del sexo, $F(1,213)=7,01 p<.01, \eta p 2=0,032$ donde los hombres tuvieron puntuaciones más altas que las mujeres, también se observaron diferencias significativas respecto del PCA, $F(2,213)=3,89 \mathrm{p}<.05, \eta p 2=0,035$. Finalmente, se encontró un efecto significativo de la interacción de sexo y PCA, F (2,213) = 3,308 p<.05, np2= 0,030 (Figura 5). El análisis post hoc mostró que, entre los hombres intensivos y los hombres pesados hay una diferencia significativa $(p<.01)$, siendo los consumidores pesados los que tienen un puntaje más alto. Por otro lado, se encontró que las mujeres con consumo intensivo puntúan más alto que las mujeres con consumo liviano $(p<.01)$.

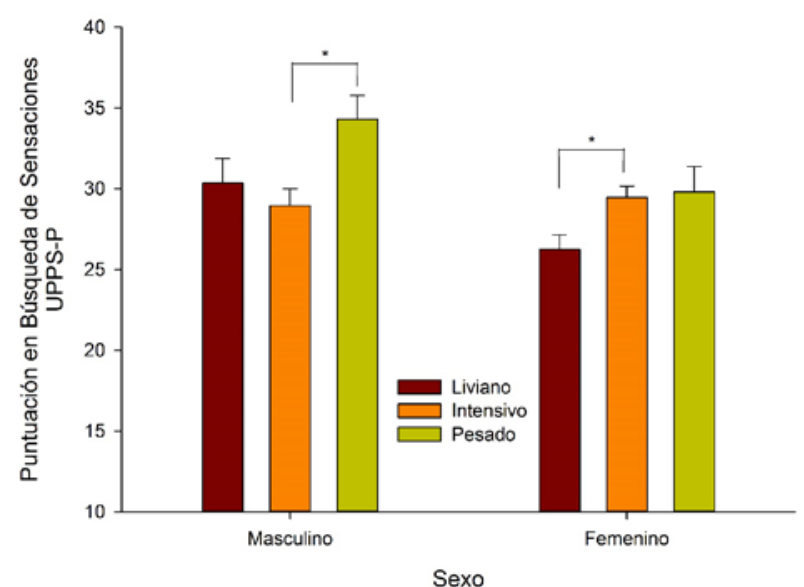

Figura 5. Puntuación en Búsqueda de Sensaciones (UPPS-P) según PCA y Sexo

Finalmente, en relación a la dimensión Urgencia Positiva, se encontró un efecto principal del PCA F $(2,213)$ $=15,472 \mathrm{p}<.001, \eta p 2=0,13$. El análisis post hoc mostró que los bebedores livianos tuvieron puntuaciones significativamente más bajas que los bebedores intensivos $(p<.001)$ y que los bebedores pesados $(p<.001)$.

\section{DISCUSIÓN Y CONCLUSIONES}

En este estudio se buscó determinar el efecto que tiene el Patrón de Consumo de Alcohol (PCA) sobre el rendimiento en pruebas de TD y FE en jóvenes de 18 a 30 años. Para determinar el PCA se utilizó un Análisis de Clases Latentes, el cual permitió identificar 3 modalidades de consumo distintas, bebedores Livianos, Intensivos y Pesados. Así, en primer lugar, el análisis de Ios puntajes en la WCST permitió observar una diferencia en la cantidad de respuestas correctas en relación al PCA. Estos resultados no son consistentes con los reportados en la literatura (ver, por ejemplo: Johnson et al., 2008), ya que los bebedores livianos hicieron menos respuestas correctas que los bebedores intensivos. Sin embargo, guardan relación con un estudio local previo (Martínez \& Manoiloff, 2010), en el que no se encontraron diferencias entre los PCA considerados con respecto a los distintos puntajes de la WCST. Al respecto, debe considerarse que se ha puesto en duda la especificidad anatómica de la WCST, ya que demanda un conjunto de procesos que están distribuidos en todo el cerebro (Nyhus \& Barceló, 2009). Por ejemplo, uno de los componentes de la WCST es el "cambio cognitivo" que requeriría de la activación de la corteza prefrontal dorsolateral. Entonces, para poder cumplir con las demandas de cambio cognitivo en la WCST, sería necesario un adecuado funcionamiento de esta estructura. Sin embargo, esta área del cerebro por sí misma no sería suficiente para dar cuenta de todos los procesos neurocognitivos implicados en su resolución (Barceló, 2001; Nyhus \& Barceló, 2009). Además, se ha sugerido que algunos de los índices con los que se mide el rendimiento con la WCST también deberían ser revisados (Nyhus \& Barceló, 2009). Tomadas en conjunto, estas críticas a la utilidad de la WCST, explicarían su baja sensibilidad para detectar diferencias que pudieran atribuirse a los distintos tipos de PCA de los participantes de este estudio.

Por otro lado, se encontró un efecto significativo del PCA en el rendimiento en la GDT. Como se esperaba, los participantes con un PCA más severo rindieron peor. Estos resultados podrían corresponderse con lo planteado por Kim, Sohn, \& Jeong, (2011), quienes reportaron que adictos y pacientes con lesiones en la corteza prefrontal llevaron a cabo elecciones sistemáticamente más desventajosas. Aunque en este estudio no se trabajó con una muestra clínica, los cambios en el SNC producidos por un PCA Pesado podrían explicar el deterioro observado.

Del mismo modo, al analizar el rendimiento en la CBT los participantes con PCA Pesado mostraron un patrón de elecciones más independiente del contexto cognitivo al compararlos con los participantes con un PCA Liviano e Intensivo. Si bien el patrón de respuestas cambió a lo largo del desempeño en la prueba, se pudo observar que los participantes con un PCA Pesado demoraron más que los otros en tomar decisiones 
usando el contexto cognitivo. Este patrón de elección coincide con lo reportado en otros estudios con consumidores de sustancias y pacientes con lesiones frontales. En efecto, el rendimiento de estos grupos de participantes es significativamente más independiente del contexto, lo que implica que la TD se basa en atributos perceptuales fijos e inmodificables, sin consideración de los aspectos relevantes del contexto de decisión en el que se encuentra el individuo (Verdejo-García, Vilar-López, Pérez-García, Podell, \& Goldberg, 2006).

En relación a las distintas subescalas de la UPPS-P, se encontraron resultados que coinciden con lo reportado por la literatura (Bechara, 2005; Bechara \& Van der Linden, 2005) y lo que se esperaba. Esto es, los participantes con un PCA más severo (Intensivo y Pesado) tuvieron puntuaciones más altas en las dimensiones de Urgencia (negativa y positiva) así como en la Falta de Premeditación y Falta de Perseverancia. Además, en el caso de la dimensión Búsqueda de Sensaciones se encontró una interacción entre sexo y PCA. Las características de la impulsividad rasgo de los participantes con un PCA más severo permiten pensar en el papel que juega la impulsividad en el establecimiento y mantenimiento de patrones de consumo de alcohol y otras sustancias que son perjudiciales para la salud (de Wit, 2009). Además, estos datos complementan reportes locales previos. En el caso de la Urgencia Positiva, en el estudio de Caña et al., (2015) se la consideró como una variable independiente $y$, junto con el PCA de los participantes, sirvió para evidenciar diferencias en el rendimiento en la TD de los participantes. Por otro lado, debido a que estas dos dimensiones tienen características emocionales intrínsecas, los resultados reportados en este trabajo van en la misma línea abordada por Michelini et al., (2016) en donde se encontró que el estado de activación emocional, tanto positiva como negativa, tuvo un efecto modulador en la TD de los participantes.

Finalmente, en este estudio no se encontraron diferencias significativas en la puntuación de la IGT (total y por bloques) en función del sexo y del PCA. Al respecto, cabe señalar que, en relación al sexo, la literatura refleja contradicciones. Por ejemplo, Bolla, Eldreth, Matochik, \& Cadet (2004) encontraron diferencias atribuibles al sexo mientras que, por otro lado, Goudriaan et al. (2007) no hallaron efectos atribuibles a esa variable sobre el desempeño en la IGT. En estudios locales previos tampoco se reportaron diferencias en función del sexo cuando se consideraron las puntuaciones de esa prueba (Bosch et al., 2016). Por otro lado, en relación al PCA de los participantes, los estudios que reportan diferencias en el rendimiento en la IGT son aquellos que compararon participantes abstemios con bebedores, principalmente bebedores intensivos (Johnson et al., 2008; Xiao et al., 2013). En estudios locales, por otro lado, solo se compararon bebedores con distinto PCA, sin un grupo de abstemios. Ello podría explicar que no se encontraran diferencias (Caña et al., 2015; Michelini et al., 2016).

Para concluir, es importante señalar como limitación de este estudio haber trabajado con una muestra de estudiantes universitarios, lo que limita la posibilidad de generalizar los resultados a jóvenes con otras características de personalidad y educativas (Henrich, Heine, \& Norenzayan, 2010). Sin embargo, haber utilizado un Análisis de Clases Latentes para la clasificación de los distintos tipos de bebedores de la muestra resulta interesante ya que permitió obtener varios niveles de clasificación y mayor sensibilidad en la detección de diferencias en la medida de impulsividad y en el rendimiento en pruebas de TD. Esto coincide con reportes previos donde se destaca la utilidad de esta herramienta en el campo de los estudios sobre el consumo de sustancias y la clasificación de tipologías de comportamiento (Reyna \& Brussino, 2011). Así, este estudio suma evidencia a la discusión sobre el papel que tiene el consumo de alcohol sobre el funcionamiento neurocognitivo en población no clínica, pero con patrones de consumo de alcohol característicos. En el mismo sentido, aporta evidencia para seguir revisando la relación propuesta entre la impulsividad y el consumo de alcohol ya sea como un determinante (ver, por ejemplo: Caña et al., 2015), o como en el presente estudio, como una consecuencia del consumo, en consonancia con las discusiones actuales sobre la relación que se puede plantear entre estas dos variables (de Wit, 2009).

\section{REFERENCIAS}

Acuña, I., Castillo, D., Bechara, A., \& Godoy, J. C. (2013). Toma de decisiones en adolescentes: rendimiento bajo diferentes condiciones de información e intoxicación alcohólica. International Journal of Psychology and Psychological Therapy, 13(2), 195-214.

Albert, D., \& Steinberg, L. (2011). Judgment and decision making in adolescence. Journal of Research on Adolescence, 21(1), 211-224. http://doi.org/10.1111/j.1532-7795.2010.00724.x 
Arnett, J. (2000). Emerging adulthood: A theory of development from the late teens through the twenties. American Psychologist, 55(5), 469480. http://doi.org/10.1037//0003-066X.55.5.469

Aron, A. (2007). The Neural Basis of Inhibition in Cognitive Control. The Neuroscientist, 13(3), 214-228. http://doi.org/10.1177/1073858407299288

Barceló, F. (2001). Does the Wisconsin Card Sorting Test Measure Prefrontal Function? The Spanish Journal of Psychology, 4(1), 79-100.

Bechara, A. (2005). Decision making, impulse control and loss of willpower to resist drugs: a neurocognitive perspective. Nature Neuroscience, 8(11), 1458-1463. http://doi.org/10.1038/nn1584

Bechara, A., Damasio, A., Damasio, H., \& Anderson, S. (1994). Insensitivity to future consequences following damage to human prefrontal cortex. Cognition, 50(1-3), 7-15.

Bechara, A., \& Van der Linden, M. (2005). Decision-Making and Impulse Control after Frontal Lobe Injuries. Current Opinion in Neurology, 18(6), 734-739.

Blakemore, S.-J., \& Robbins, T. (2012). Decision-making in the adolescent brain. Nature Neuroscience, 15(9), 1184-91. http://doi.org/10.1038/nn.3177

Bolla, K., Eldreth, D., Matochik, J., \& Cadet, J. (2004). Sex-related differences in a gambling task and its neurological correlates. Cerebral Cortex, $14,1226-1232$

Bosch, C., Miranda, J., Sangiorgio, M., Acuña, I., Michelini, Y., Marengo, L., \& Godoy, J. C. (2016). Efecto del entrenamiento con un juego de estrategia en tiempo real sobre la toma de decisiones en adolescentes. Psiencia, Revista Latinoamericana de Ciencia Psicológica, 8(1), 1-19. http://doi.org/10.5872/psiencia/8.1.22

Brand, M., Fujiwara, E., Borsutzky, S., Kalbe, E., Kessler, J., \& Markowitsch, H. (2005). Decision-making deficits of korsakoff patients in a new gambling task with explicit rules: associations with executive $\begin{array}{llll}\text { functions. } & \text { 26uropsychology, }\end{array}$ http://doi.org/10.1037/0894-4105.19.3.267

Caña, M. L., Michelini, Y., Acuña, I., \& Godoy, J. C. (2015). Efectos de la impulsividad y el consumo de alcohol sobre la toma de decisiones en los adolescentes. Health and Addictions/Salud Y Drogas, 15(1), 5566.

Casey, B., Getz, S., \& Galván, A. (2008). The adolescent brain. Developmental Review, 28(1), 62-77. http://doi.org/10.1016/j.dr.2007.08.003

Coskunpinar, A., Dir, A. L., \& Cyders, M. (2013). Multidimensionality in Impulsivity and Alcohol Use: A Meta-Analysis Using the UPPS Model of Impulsivity. Alcoholism: Clinical and Experimental Research, 37(9), 1441-1450. http://doi.org/10.1111/acer.12131

Crews, F., He, J., \& Hodge, C. (2007). Adolescent cortical development: A critical period of vulnerability for addiction. Pharmacology, Biochemistry and Behavior, 86(2), 189-199. http://doi.org/10.1016/j.pbb.2006.12.001

Cyders, M. (2013). Impulsivity and the sexes: measurement and structural invariance of the UPPS-P Impulsive Behavior Scale. Assessment, 20(1), 86-97. http://doi.org/10.1177/1073191111428762

de Wit, H. (2009). Impulsivity as a determinant and consequence of drug use: a review of underlying processes. Addiction Biology, 14(1), 2231. http://doi.org/10.1111/j.1369-1600.2008.00129.x
Eysenck, H. J., \& Eysenck, S. B. G. (1997). Cuestionario Revisado de Personalidad de Eysenck (EPQ-R). Manual [Eysenck Personality Questionnaire Revised (EPQ-R). Manual]. Madrid: Tea Ediciones, S.A.

Goldberg, E., Harner, R., Lovell, M., Podell, K., \& Riggio, S. (1994). Cognitive bias, functional cortical geometry and the frontal lobes: laterality, sex and handedness. Journal of Cognitive Neuroscience, 6(3), 276296.

Goldstein, R. Z., \& Volkow, N. D. (2002). Drug Addiction and Its Underlying Neurobiological Basis: Neuroimaging Evidence for the Involvement of the Frontal Cortex. American Journal of Psychiatry, 159(October), 1642-1652.

Goudriaan, A., Grekin, E. R., \& Sher, K. (2007). Decision Making and Binge Drinking: A Longitudinal Study. Alcoholism: Clinical and Experimental Research, 31(6), 928-938. http://doi.org/10.1111/j.15300277.2007.00378.x

Grant, D. A., \& Berg, E. A. (1948). A behavioral analysis of degree of reinforcement and ease of shifting to new responses in a Weigl-type card-sorting problem. Journal of Experimental Psychology, 38(4), 404411

Hall, G. S. (1904). Adolescence: Its psychology and its relations to physiology, anthropology, sociology, sex, crime, religion, and education. New York: Appleton.

Hanson, K. L., Medina, K. L., Padula, C. B., Tapert, S., \& Brown, S. (2011). Impact of Adolescent Alcohol and Drug Use on Neuropsychological Functioning in Young Adulthood: 10-Year Outcomes. Journal of Child \& Adolescent Substance Abuse, 20(2), 135-154. http://doi.org/10.1080/1067828X.2011.555272

Heaton, R., Chelune, G., Talley, J., Kay, G., \& Curtis, G. (1993). Wisconsin Card Sorting Test Manual (1st ed.). Lutz: Psychilogical Assessment Resources.

Henrich, J., Heine, S. J., \& Norenzayan, A. (2010). The weirdest people in the world? The Behavioral and Brain Sciences, 33, 1-23. http://doi.org/10.1017/S0140525X0999152X

Johnson, C. A., Xiao, L., Palmer, P., Sun, P., Wang, Q., Wei, Y. \& Bechara, A. (2008). Affective decision-making deficits, linked to a dysfunctional ventromedial prefrontal cortex, revealed in 10th grade Chinese adolescent binge drinkers. Neuropsychologia, 46, 714-726. http://doi.org/10.1016/j.neuropsychologia.2007.09.012

Kim, Y.-T., Sohn, H., \& Jeong, J. (2011). Delayed transition from ambiguous to risky decision making in alcohol dependence during lowa Gambling Task. Psychiatry Research, 190(2-3), 297-303. http://doi.org/10.1016/j.psychres.2011.05.003

Littlefield, A., Sher, K. J., \& Steinley, D. (2010). Developmental trajectories of impulsivity and their association with alcohol use and related outcomes during emerging and young adulthood I. Alcoholism: Clinical and Experimental Research, 34(8), 1409-1416. http://doi.org/10.1111/j.1530-0277.2010.01224.x

Martínez-Selva, J. M., Sánchez-Navarro, J. P., Bechara, A., Román, F., (2006). Mecanismos cerebrales de la toma de decisiones. Revista de Neurología, 42(7), 411-418.

Martínez, M. V., \& Manoiloff, L. M. V. (2010). Evaluación Neuropsicológica de la Función Ejecutiva en Adolescentes con diferentes patrones de consumo de alcohol. Revista Argentina de Ciencias Del Comportamiento, 2(1), 14-23. 
Michelini, Y., Acuña, I., \& Godoy, J. C. (2016). Emociones, toma de decisiones y consumo de alcohol en jóvenes universitarios. Suma Psicológica, 23, 42-50. http://doi.org/10.1016/j.sumpsi.2016.01.001

Montero, I., \& León, O. G. (2007). A guide for naming research studies in Psychology. International Journal of Clinical and Health Psychology, 7(3), 847-862.

Mota, N., Parada, M., Crego, A., Doallo, S., Caamaño-Isorna, F., Rodríguez Holguín, S. \& Corral-Varela, M. (2013). Binge drinking trajectory and neuropsychological functioning among university students: a longitudinal study. Drug and Alcohol Dependence, 133(1), 108-14. http://doi.org/10.1016/j.drugalcdep.2013.05.024

National Institute on Alcohol Abuse and Alcoholism. (2013). Alcohol Overdose: The Dangers of Drinking Too Much. National Institute on Alcohol Abuse and Alcoholism.

Nyhus, E., \& Barceló, F. (2009). The Wisconsin Card Sorting Test and the cognitive assessment of prefrontal executive functions: A critical update. Brain and Cognition, 71(3), 437-451. http://doi.org/10.1016/j.bandc.2009.03.005

Organización Mundial de la Salud. (2014). Global status report on alcohol and health 2014. Global status report on alcohol. Luxemburg. Retrieved

from http://www.who.int/substance_abuse/publications/global_alcohol report/msbgsruprofiles.pdf

Parada, M., Corral-Varela, M., Caamaño-Isorna, F., Mota, N., Crego, A., Rodríguez Holguín, S. \& Cadaveira, F. (2011). Definición del concepto de consumo intensivo de alcohol adolescente (binge drinking). Adicciones, 23(1), 53-63.

Parada, M., Corral-Varela, M., Mota, N., Crego, A., Holguín, S. R., \& Cadaveira Mahía, F. (2012). Executive functioning and alcohol binge drinking in university students. Addictive Behaviors, 37(2), 167-172. http://doi.org/10.1016/j.addbeh.2011.09.015

Pilatti, A., Brussino, S., \& Godoy, J. C. (2013). Factores que influyen en el consumo de alcohol de adolescentes argentinos: un path análisis prospectivo. Revista de Psicología, 22(1), 22-36. http://doi.org/10.5354/0719-0581.2013.27716

Pilatti, A., Caneto, F., Garimaldi, J. A., Vera, B. del V., \& Pautassi, R. M. (2014). Contribution of time of drinking onset and family history of alcohol problems in alcohol and drug use behaviors in argentinean college students. Alcohol and Alcoholism, 49(2), 128-137. http://doi.org/10.1093/alcalc/agt176

Pilatti, A., Godoy, J. C., Brussino, S., \& Pautassi, R. M. (2013). Underage drinking: Prevalence and risk factors associated with drinking experiences among Argentinean children. Alcohol, 47(4), 323-31. http://doi.org/10.1016/j.alcohol.2013.02.001

Quinn, P. D., \& Harden, K. P. (2013). Differetial Changes in impulsivity and sensation seeking and the escalation of substance use from adolescence to early adulthood. Developmental Psychopathology, 25(1), 223-239. http://doi.org/10.1017/S0954579412000284.Differential

Reyna, C., \& Brussino, S. (2011). Revisión de los fundamentos del análisis de clases latentes y ejemplo de aplicación en el área de las adicciones. Trastornos Adictivos, 13(1), 11-19.

Rogers, R. D., Moeller, F. G., Swann, A. C., \& Clark, L. (2010). Recent research on impulsivity in individuals with drug use and mental health disorders: Implications for alcoholism. Alcoholism: Clinical and
Experimental Research, 34(8),

http://doi.org/10.1111/j.1530-0277.2010.01216.x

Shulman, E., Smith, A. R., Silva, K., Icenogle, G., Duell, N., Chein, J., \& Steinberg, L. (2016). The dual systems model: Review, reappraisal, and reaffirmation. Developmental Cognitive Neuroscience, 17, 103117. http://doi.org/10.1016/j.dcn.2015.12.010

Spear, L. (2013). Adolescent Neurodevelopment. Journal of Adolescent Health, 52(2), S7-S13. http://doi.org/10.1016/j.jadohealth.2012.05.006

Spear, L. (2015). Adolescent alcohol exposure: Are there separable vulnerable periods within adolescence? Physiology \& Behavior, 148, 122-30. http://doi.org/10.1016/j.physbeh.2015.01.027

Stautz, K., \& Cooper, A. (2013). Impulsivity-related personality traits and adolescent alcohol use: A meta-analytic review. Clinical Psychology Review, 33(4), 574-592. http://doi.org/10.1016/j.cpr.2013.03.003

Steinberg, L. (2008). A social neuroscience perspective on adolescent risktaking. Developmental Review, 28(1), 1-27.

Tabachnick, B., \& Fidell, L. (2007). Using Multivariate Statistics (5th ed.) Boston: Pearson Education.

Verdejo-García, A., Lozano, Ó., Moya, M., Alcázar, M. Á., \& Pérez-García, M. (2010). Psychometric properties of a Spanish version of the UPPS-P impulsive behavior scale: reliability, validity and association with trait and cognitive impulsivity. Journal of Personality Assessment, 92(1), 70-7. http://doi.org/10.1080/00223890903382369

Verdejo-García, A., Vilar-López, R., Pérez-García, M., Podell, K., \& Goldberg, E. (2006). Altered adaptive but not veridical decision-making in substance dependent individuals. Journal of the International Neuropsychological Society, 12(1), 90-99. http://doi.org/10.1017/S1355617706060127

White, H. R., Marmorstein, N. R., Crews, F., Bates, M. E., Mun, E., \& Loeber, R. (2011). Associations Between Heavy Drinking and Changes in Impulsive Behavior Among Adolescent Boys. Alcoholism: Clinical and Experimental Research, 35(2), 1-9. http://doi.org/10.1111/j.1530$\underline{0277.2010 .01345 . x}$

Wood, P. K., Sher, K., \& Rutledge, P. C. (2007). College Student Alcohol Consumption, Day of the Week, and Class Schedule. Alcoholism: Clinical and Experimental Research, 31(7), 1195-1207. http://doi.org/10.1111/j.1530-0277.2007.00402.x

Xiao, L., Bechara, A., Gong, Q., Huang, X., Li, X., Xue, G., ... Johnson, C. A. (2013). Abnormal affective decision making revealed in adolescent binge drinkers using a functional magnetic resonance imaging study. Psychology of Addictive Behaviors, 27(2), 443-54. http://doi.org/10.1037/a0027892 